\title{
Multimodal Interaction in Psychopathology
}

\author{
Itir Onal Ertugrul \\ Department of Cognitive Science and \\ Artificial Intelligence \\ Tilburg University \\ Tilburg, The Netherlands \\ i.onal@uvt.nl
}

\author{
Jeffrey F. Cohn \\ Department of Psychology \\ University of Pittsburgh \\ Pittsburgh, PA, USA \\ jeffcohn@pitt.edu
}

\author{
Hamdi Dibeklioglu \\ Department of Computer Engineering \\ Bilkent University \\ Ankara, Turkey \\ dibeklioglu@cs.bilkent.edu.tr
}

\begin{abstract}
This paper presents an introduction to the Multimodal Interaction in Psychopathology workshop, which is held virtually in conjunction with the 22nd ACM International Conference on Multimodal Interaction on October 25th, 2020. This workshop has attracted submissions in the context of investigating multimodal interaction to reveal mechanisms and assess, monitor, and treat psychopathology. Keynote speakers from diverse disciplines present an overview of the field from different vantages and comment on future directions. Here we summarize the goals and the content of the workshop.
\end{abstract}

\section{CCS CONCEPTS}

- Human-centered computing;

\section{KEYWORDS}

psychopathology, multimodal intra- and interpersonal behavior, brain activity

ACM Reference Format:

Itir Onal Ertugrul, Jeffrey F. Cohn, and Hamdi Dibeklioglu. 2020. Multimodal Interaction in Psychopathology. In 2020 International Conference on Multimodal Interaction (ICMI '20), October 25-29, 2020, Virtual Event, Netherlands. ACM, New York, NY, USA, 2 pages. https://doi.org/10.1145/3382507.3419751

\section{INTRODUCTION}

Millions of people worldwide are affected by mental disorders that include unipolar and bipolar depression, obsessive-compulsive disorder, schizophrenia, and dementia. Reliable assessment, monitoring, and evaluation and understanding of mechanisms are essential to identify individuals in need of treatment, evaluate treatment response, and achieve remission or moderate impact. Many indicators of presence or severity of mental disorders are observable. Indicators include psychomotor agitation (inability to sit still, pacing, hand wringing) or retardation (slowed speech and body movements, speech that is decreased in volume or vocal quality), changes in facial expression, gaze, body movements, and cognition. Attempts at diagnosis, screening and evaluation of treatment response from behavioral indicators have focused primarily on the individual alone

Permission to make digital or hard copies of all or part of this work for personal or classroom use is granted without fee provided that copies are not made or distributed for profit or commercial advantage and that copies bear this notice and the full citation on the first page. Copyrights for components of this work owned by others than ACM must be honored. Abstracting with credit is permitted. To copy otherwise, or republish, to post on servers or to redistribute to lists, requires prior specific permission and/or a fee. Request permissions from permissions@acm.org.

ICMI '20, October 25-29, 2020, Virtual Event, Netherlands

(C) 2020 Association for Computing Machinery.

ACM ISBN 978-1-4503-7581-8/20/10...\$15.00

https://doi.org/10.1145/3382507.3419751 and individual modalities. Yet, disorders strongly impact social interaction and relationships in family members, work settings, and on social media and are multimodal as well as interpersonal. For these reasons, it is critical to use multimodal indicators in a variety of interpersonal contexts.

The proposed Multimodal Interaction in Psychopathology workshop aims to bring together computer scientists, psychologists, behavioral scientists, neuroscientists, and clinicians with a focus on multimodal interaction in psychopathology. This workshop provides an opportunity to present recent advancements in the understanding and diagnosis and treatment of mental disorders, to share knowledge, and generate interdisciplinary networking and collaborations.

\section{WORKSHOP CONTENT}

Research submitted to the workshop covers a large range of topics centered on investigating multimodal interaction for the analysis, assessment, monitoring, and treatment of psychopathology. Some topics that are focused in the submissions are ambient pain monitoring of older adults, social communication behaviors of children, discrepant mother-infant face-to-face communication in risk contexts, deep brain stimulation for treatment-resistant obsessivecompulsive disorder (OCD), emotion streams for mental health surveillance, and multimodal measurement of classroom interaction. Mental disorders addressed in the submissions include autism spectrum disorder (ASD), OCD, dementia, and maternal anxiety and depression. Models and analyses proposed in these papers employ diverse modalities including brain activity, facial expression, face \& head dynamics, gaze, facial and vocal affect, spatial orientation, and touch. Ages of interest span life course from infancy, childhood, and early through late adulthood.

The workshop is enriched by the following talks of the six invited speakers:

- Modeling Speech in the Real World: Detecting Mood Severity via Affective Computing by Emily Mower Provost, University of Michigan

- Smartphone mobile sensing as a diagnostic and therapeutic tool: The case of suicide prediction and prevention by Nick Allen, University of Oregon

- Building classifiers for adaptive deep brain stimulation for $O C D$ by Wayne Goodman, Baylor College of Medicine

- Data Drive Development-Objective Measurement of Early Interaction by Daniel Messinger, University of Miami 
- All communication modalities illuminate risk in the 4-month mother-infant origins of 12-month infant disorganized attachment by Beatrice Beebe, Columbia University and New York State Psychiatric Institute

- Linking facial expression and brain activity to understand learning in children's development by Nathan Fox, University of Maryland, College Park

\section{WORKSHOP ORGANIZATION}

\subsection{Organizing Committee}

- Itir Onal Ertugrul, Tilburg University

- Jeffrey F. Cohn, University of Pittsburgh

- Hamdi Dibeklioglu, Bilkent University

\subsection{Program Committee}

- Abhinav Dhall, Monash University

- Jyoti Joshi Dhall, Monash University

- Sergio Escalera, University of Barcelona

- Jeffrey Girard, Carnegie Mellon University

- Laszlo Jeni, Carnegie Mellon University

- Dongmei Jiang, Northwestern Polytechnical University

- Heysem Kaya, Utrecht University

- Albert Ali Salah, Utrecht University
- Juan Pablo Wachs, Purdue University

- Lijun Yin, Binghamton University

\subsection{Review process}

All of the accepted papers and extended abstracts received two or more double-blind reviews. Reviewers consisted of the invited program committee members who have diverse expertise. Papers were randomly assigned to reviewers.

\section{CONCLUSION}

The range of submissions shows that multimodal interaction in psychopathology attracts psychologists and other behavioral scientists, psychiatrists, and computer scientists. An interdisciplinary perspective is critical to the field. By providing an environment for bringing researchers from across disciplines to develop further multidisciplinary collaborations, this workshop contributes to advancing this field.

\section{ACKNOWLEDGMENTS}

We would like to thank the program committee members for their invaluable contribution during the review process. Additional support was provided by U.S. National Institutes of Health award MH096951 and U.S. National Science Foundation award IIS-1721667. 